\title{
Arborescent Bacterial Keratopathy (Infectious Crystalline Keratopathy)
}

\author{
A. P. WATSON*, A. B. TULLO*, M. G. KERR-MUIR $†$, A. E. A. RIDGWAY* and \\ D. R. LUCAS§
}

Manchester and London

\begin{abstract}
Summary:
Bacterial colonisation of the cornea is described in two cases, one bilateral. Discreet, white, branching opacities are produced without associated inflammation. It is suggested that due to its aetiology "arborescent bacterial keratopathy" is a better name for this condition than "infectious crystalline keratopathy".
\end{abstract}

Infectious crystalline keratopathy is a recently identified corneal disorder which has been described in several patients in the USA $^{1-9}$. The condition occurs in corneae which have been treated with topical steroids over a long period. The majority of patients reported had undergone a penetrating keratoplasty ${ }^{1-7}$. Other predisposing conditions are Herpes simplex keratitis ${ }^{3}$ and Ácanthamoeba keratitis $8,10,11$. The characteristic appearance is of a white, feathery, crystalline deposit, without associated inflammation, in the anterior corneal stroma. Viridans streptococci have been the organisms most frequently identified within affected corneae by culture and electron microscopy ${ }^{1-6}$. We describe 2 patients, one affected bilaterally, who developed this condition as a complication of penetrating keratoplasty.

\section{Case Reports}

Patient 1:

An otherwise healthy 31 year old woman with bilateral lens opacities underwent left extracapsular cataract surgery, followed one year later by a right extracapsular cataract extraction complicated by rupture of the posterior capsule with vitreous loss. Gradual corneal decompensation followed, and bilateral penetrating keratoplasties were performed 12 years after her cataract surgery. Both eyes were treated with topical betamethasone sodium phosphate $0.5 \%$ three times a day (also atropine $1 \%$ and phenylephrine $10 \%$ ) continuously, apart from a one month period, from the time of surgery until the patient was referred to our hospital for assessment 6 months later.

The visual acuity was hand movements only in either eye. Both grafts were decompensated, with continuous monofilament nylon sutures in place. In the left graft there was an area of white, branching opacities within the corneal stroma, but with no associated infiltrate (Fig 1). A few keratic precipitates could be seen. The intraocular pressure was normal. Treatment with topical steroids was continued. An $8 \mathrm{~mm}$ penetrating graft was performed ten months after the first graft. The postoperative period was complicated by anterior uveitis lasting five months, and raised intraocular pressure. Betamethasone drops were used up to four times a day for five months.

Histopathological examination of the excised left corneal button showed clusters of basophilic particles within the corneal stroma. Electron microscopy revealed cocci with a laminated cell wall, consistent with a diagnosis of streptococcal colonisation (Fig. 2).

From *Manchester Royal Eye Hospital.

$\S$ Departments of Ophthalmology and Pathology, University of Manchester.

†St Thomas' Hospital, London.

Correspondence to: Manchester Royal Eye Hospital, Oxford Road, Manchester. 
Two years after the repeat graft to the left eye an area of crystalline deposits was noted within the stroma of the decompensated right corneal graft. The right eye had been treated almost continuously with topical steroids (betamethasone or prednisolone). As the appearance was similar to that of the left corneal graft prior to its replacement, treatment was begun with 2-hourly penicillin drops. The lesion continued to expand with the advancing edge maintaining a linear, feathery, crystalline appearance. The area where the lesion

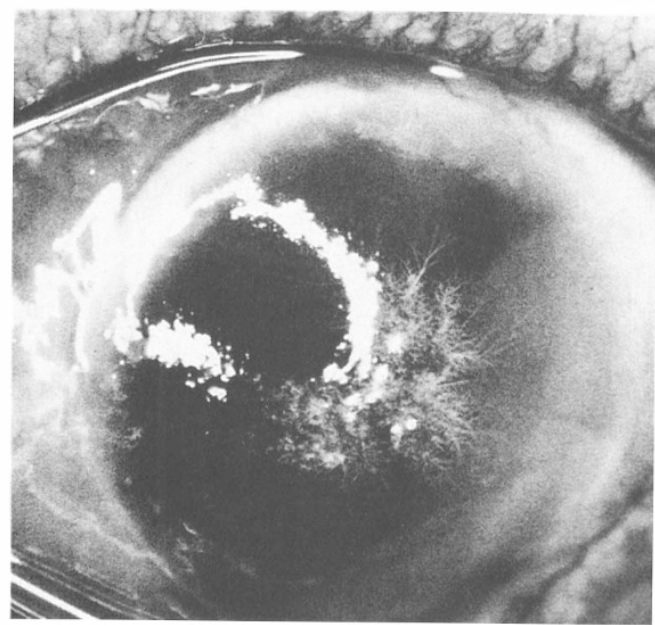

Fig. 1. The left eye of patient 1 showing typical arborescent stromal lesion.

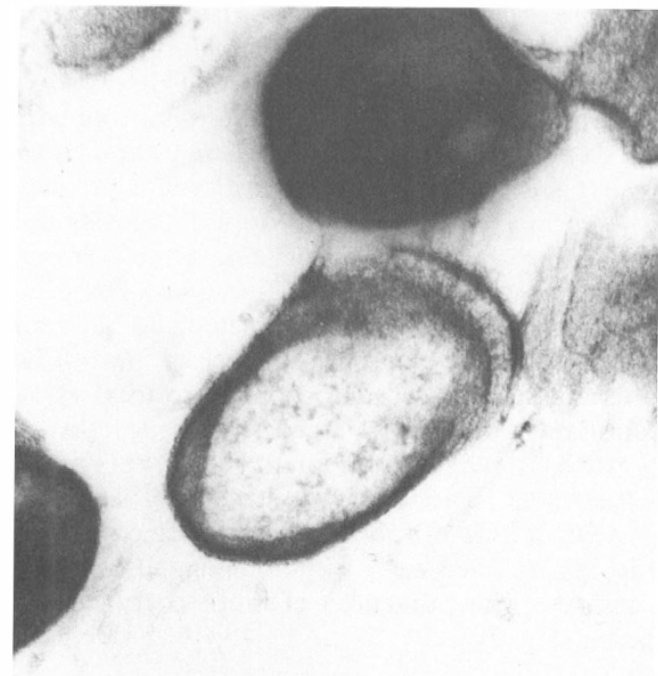

Fig. 2. (X177.5) Electron micrograph of corneal button removed from left eye of patient 1 showing cocci with laminated cell wall typical of viridans streptococci. had first been seen became amorphous and necrotic, with breakdown of the overlying epithelium. A second penetrating keratoplasty was also performed on this eye.

Histopathological examination of the excised right corneal button showed a small focus of necrosis in the stroma close to one margin. This communicated with the surface and epithelial fragments had been punched into it at surgery. There was minimal infiltration of this area with polymorphs and pyknotic debris. Adjacent to the necrotic area the stroma was extremely oedematous. Numerous clusters of cocci were identified lying between the stromal lamellae over a wide area in which no cellular reaction was apparent (Fig. 3). The cocci stained only faintly, even by Gram's method, and no organisms were grown from a cultured portion of the cornea. The appearance of the clusters of cocci between the lamellae was essentially similar to that seen in the corneal disc previously removed from the left eye, and corresponded in position to the advancing edge of the lesion which had maintained an apparently crystalline pattern on slit lamp examination.

\section{Patient 2:}

A 65 year old Caucasian man presented with deteriorating sight in each eye, having had poor vision since childhood. He had bilateral diffuse corneal scarring, due to quiescent interstitial keratitis, and cataract. Treponemal screening tests were positive for both blood and cerebrospinal fluid, and the patient had a course of intramuscular penicillin. There were no systemic or ocular complications.

He underwent a combined left cataract extraction with $8.0 \mathrm{~mm}$ penetrating keratoplasty. The donor was a 59 year old male who had died from large bowel adenocarcinoma. There was a nine hour interval between death and insertion of the whole eye into a moist storage chamber, with surgery occurring three hours later. The donor epithelium was removed prior to keratoplasty. Post-operatively the eye was treated with hourly guttae prednisolone $1 \%$ by day and 2-hourly by night, in addition to guttae chloramphenicol 0.5 qds. After two weeks the graft had reepithelialised save for a linear defect in the inferotemporal quadrant and the topical steroid was reduced to 2 hourly. Guttae timolol $0.5 \%$ bd was added as the intraocular pressure was $34 \mathrm{mmHg}$.

Four weeks post-operatively there was mild, diffuse graft oedema and although there was no epithelial defect, the site of recent epithelial apposition was still evident in the infero-temporal quadrant. The topical prednisolone $1 \%$ was reduced to 4-hourly, the chloramphenicol stop- 


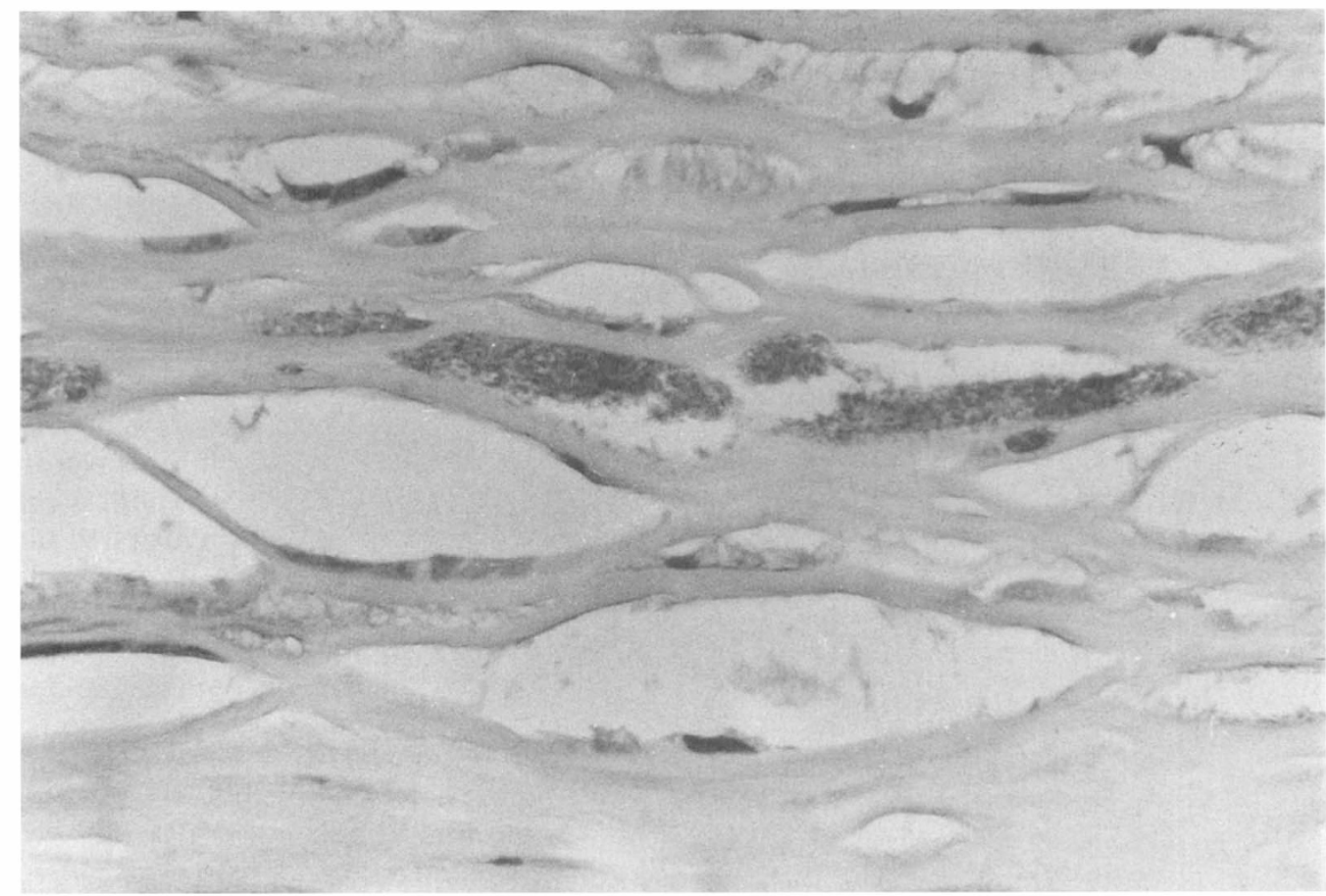

Fig. 3. (Gram X540) Corneal button from right eye of patient 1 showing typical interlamellar collections of bacteria in the anterior stroma.

ped, and with the intraocular pressure at $20 \mathrm{mmHg}$ the timolol was continued.

Seven weeks post-operatively the corrected acuity was $6 / 36$ in the presence of an intact corneal epithelium, slight folding of Descemet's membrane, but an otherwise uninflamed eye. The guttae prednisolone was reduced to qds and the timolol continued.

Three months after surgery the patient returned with a five day history of impaired vision in the left eye, in association with a mucoid discharge. The bulbar conjunctiva was moderately injected, particularly in the infero-temporal quadrant. A linear epithelial defect of $1.5 \mathrm{~mm}$ had re-appeared in the infero-temporal quadrant of the graft. Deep to this there was a dense opacity with discreet, multidirectional, cylindrical extensions into clear stroma centrally (Fig 4). A corneal scrape at the edge of the epithelial defect did not yield any micro-organisms.

The eye was treated with guttae chloramphenicol $0.5 \%$ three hourly, while the prednisolone $1 \%$ and timolol were continued as before. By the following day, whilst the bulbar conjunctival injection had not changed, there was an increase in infiltrate immediately deep to the epithelial defect. A repeat corneal scrape showed gram positive cocci, which provided a heavy growth of viridans streptococci on culture, sensitive to penicillin.

For the next two weeks the eye was treated with hourly penicillin by day, and 2-hourly by night. Although the epithelial defect remained unchanged the underlying stromal opacities began to coalesce. After another week of intensive topical penicillin the epithelium had healed with an underlying confluent anterior stromal opacity, associated with an area of corneal thinning.

\section{Discussion:}

The patients described above fulfill the criteria for diagnosis of the condition presently named "infectious crystalline keratopathy". The crystalline appearance of the deposits, without any associated inflammation, within the anterior stroma of the cornea would suggest that the condition is degenerative or dystrophic. However, it is established that it is caused by interstitial colonisation of the corneal stroma by bacteria. Viridans streptococci have been identified within the corneal stroma by electron microscopy and culture of corneal tissue. ${ }^{1-6}$ These 


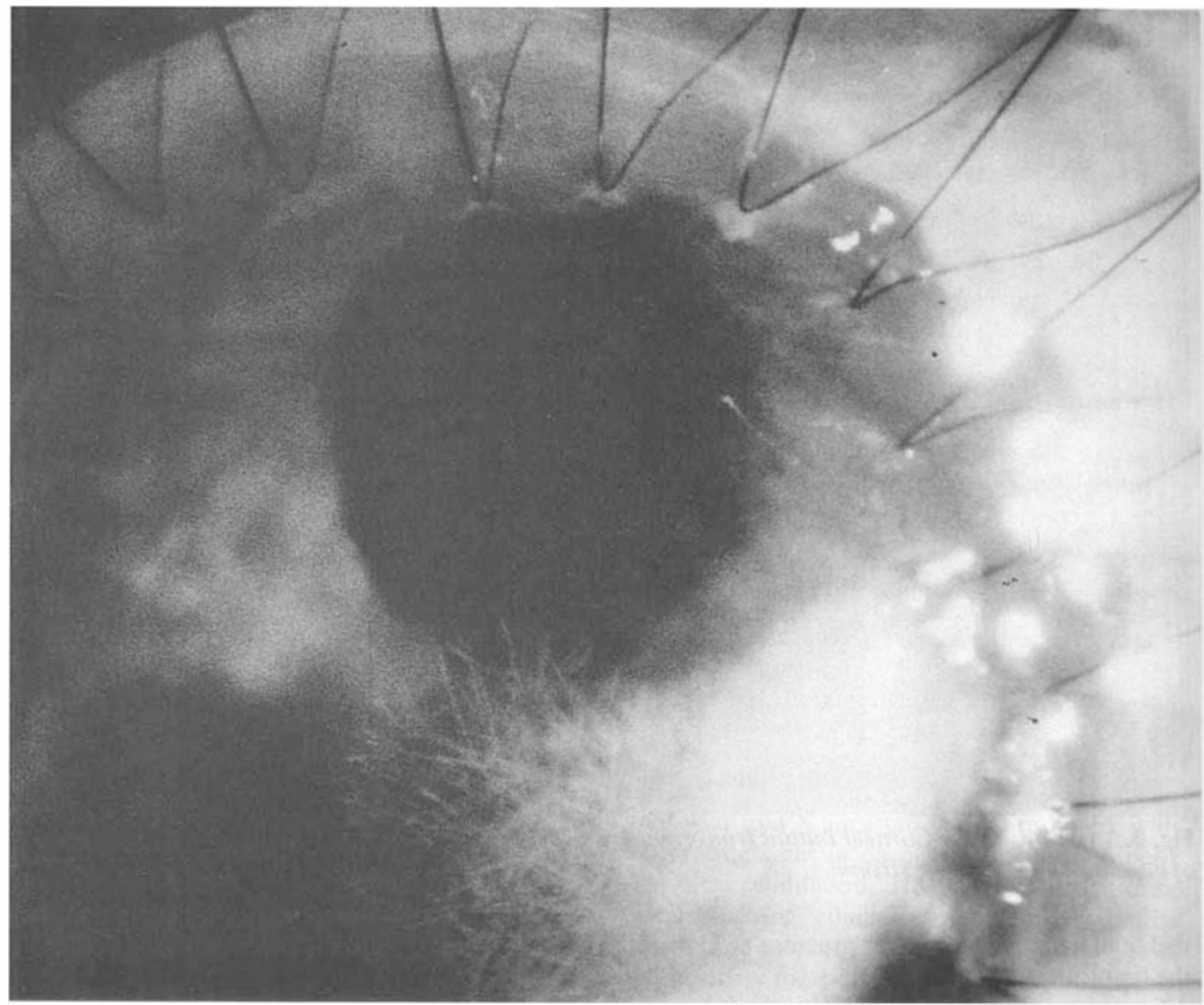

Fig. 4. Slit lamp photograph of corneal lesion in patient 2 showing dense opacity with discreet multidirectional extensions.

organisms were also found in both the patients described above. In one recent report $^{7}$ Haemophilus aphrophilus was cultured from a corneal swab from a patient with a similar keratopathy, where the typical branching lesion in the corneal stroma of an uninflamed eye developed in a corneal graft beneath a persistent epithelial defect. The anaerobic Ṕeptostreptococcus has also been cultured from the corneal button removed from an eye with a filamentous intrastromal keratitis developing after penetrating keratoplasty 9 .

Viridans streptococci are found as part of the normal flora of the oropharynx and conjunctiva. They are noncapsulate cocci with little intrinsic pathogenicity ${ }^{12}$ but which, under conditions of susceptibility, can infect tissues. The best known situation in which this can occur is bacteraemia after dental extraction leading to colonisation of an abnormal heart valve and subacute bacterial endocarditis. They can also play a major role in the formation of dental caries and are pathogens in periodontal infections. They do not have the capacity to invade the healthy or even traumatised cornea. Haemophilus aphrophilus is also a member of the normal flora of the oropharynx and is not recognised as a potential corneal pathogen. The main normal habitats of the anaerobic streptococci are the vagina and the intestine. In the majority of cases the origin of the cocci which invade a compromised cornea is probably the flora of the host conjunctiva or oropharynx, and therefore the keratopathy should not be termed "infectious", which implies transmissibility. 
The branching, crystalline appearance of the lesions is probably due to the multiplication of the bacteria along natural interlamellar lines of cleavage within the stroma. There is no true crystal formation, and therefore "crystalline" is misleading with regard to the aetiology of this condition.

All but one previously reported cases had been treated with topical steroids for an extended period. Endogenous Śtreptococcus and possibly Haemophilus bacilli apparently have the capacity to colonise the corneal stroma when it is vulnerable due to longterm application of topical steroids, as occurred in our two patients. It is unclear whether this is due to an effect on the organism itself, in a manner analogous to the enhanced replication of the Herpes simplex virus, or local immunosuppression enabling these commensal organisms to become pathogenic, or to a combination of both factors. It has been suggested that the ability of Ácanthamoeba to suppress the function of infiltrating macrophages, in addition to the immunosuppression effected by topical corticosteroids, may further enhance the development of intrastromal bacterial keratitis. ${ }^{8}$

It is unclear how bacteria gain access to the corneal stroma. The presence of epithelial downgrowth along a suture track in the first case reported in the United States of America suggested to the authors that this might have provided a route of entry for bacteria ${ }^{1}$, and it is noteworthy that bacterial colonisation first occurred in the area of previous epithelial deficiency in case 2 above. In the case of Haemophilus aphrophilus colonisation $^{7}$ also the lesion occurred beneath a persistent epithelial defect. Another possibility is that bacteria could be implanted with donor tissue at the time of surgery.

Patients with this keratopathy have been diagnosed chiefly after microscopic confirmation of the presence of streptococci in the host corneal button following penetrating keratoplasty. However, in case 2 above (and in the case of $\mathrm{H}$. aphrophilus colonisation) the diagnosis was made following culture of organisms from a corneal scrape or swab, and following intensive treatement with topical antibiotics it was not necessary to replace the grafts. Intensive topical antibiotic treatment was ineffective in controlling the keratitis in the second eye of patient 1 described above.

This keratopathy should now be kept in mind as a condition which may occur when long term topical steroid treatment is used, particularly on an eye with a compromised corneal epithelium. Although the natural history of the condition is not known, it is reasonable at present to limit topical steroids if possible, and treat with an appropriate antibiotic, though viridans streptococci are more likely to be resistant to penicillin than most other streptococci.

Although changes in nomenclature can lead to confusion, we believe that "infectious crystalline keratopathy" is not a good name for this condition, as it is not infectious and there are no crystals present. We suggest that "arborescent bacterial keratopathy" would be more appropriate as it accurately describes the appearance and the cause, and is unlikely to be confused with any other keratopathy.

\section{References}

${ }^{1}$ Gorovy MS, Stern GA, Hood CI, Allen C: Intrastromal noninflammatory bacterial colonization of a corneal graft. Arch Ophthalmol 1983, 101: 1749-52.

2 Meisler DM, Langston RHS, Naab TJ, Aaby AA, McMahon JT, Tubbs RR: Infectious corneal crystalline formation. Am J Ophthalmol 1984, 97: 337-43.

${ }^{3}$ Meisler DM, Langston RHS, Naab TJ, Aaby AA, Stern GS, Binder PS: Infectious corneal crystalline formation. Invest Ophthalmol Vis Sci (Suppl) 1984, 25: 23.

${ }^{4}$ Samples JR, Baumgartner SD, Binder PS: Infectious crystalline keratopathy: An electron microscope analysis. Cornea 1985/1986, 4: 118-26.

${ }^{5}$ Reiss GR, Campbell RJ, Bourne WM: Infectious crystalline keratopathy. Surv Ophthalmol 1986, 31: 69-72.

${ }^{6}$ Nanda M, Kaz Soong H, Krenz MP, Green WR: Intracorneal bacterial colonization in crystalline pattern. Graefe's Arch Clin Exp Ophthalmol 1986, 224: 251-5.

${ }^{7}$ Groden LR, Pascucci SE, Brinser JH: Haemophilus aphrophilus as a cause of crystalline keratopathy. Am J Ophthalmol 1987, 104: 89 . 
${ }^{8}$ Davis RM, Schroeder RP, Rowsey JJ, Jensen HG, Tripathi RC: Acanthamoeba keratitis and infectious crystalline keratopathy. Arch Ophthalmol 1987, 105: 1524-7.

${ }^{9}$ Eiferman RA, Ogden LL, Snyder J: Anaerobic Peptostreptococcal keratitis. Am J Ophthalmol 1985, 100: 335-6.

${ }^{10}$ Mathers W, Stevens G Jr, Rodrigues $M$ et al: Immunopathology and electron microscopy of
Acanthamoeba keratitis. Am J Ophthalmol 1984, 97: 337-43.

${ }^{11}$ Cohen EJ,Parlato CJ, Arentsen JJ et al: Medical and surgical treatment of Acanthamoeba keratitis Am J Ophthalmol 1987, 103: 615-25.

${ }^{12}$ Lennette EH, Balows A, Hausler WJ, Shadomy HJ (eds): Manual of clinical microbiology, 4th edition. Ch $16 \mathrm{p}$ 156. American sociey for microbiology, Washington. 1985. 